\title{
Functional relevance of a non-synonymous substitution in the CD5 gene (V471A) targeted for positive selection in East Asian populations
}

\author{
T Bielig $^{1^{*}}$, E Carnero-Montoro ${ }^{2}$, L Bonet ${ }^{2}$, J Engelken ${ }^{2}$, M Arias $^{1}$, E Bosch², F Lozano ${ }^{1,3}$ \\ From 5th European Workshop on Immune-Mediated Inflammatory Diseases \\ Sitges-Barcelona, Spain. 1-3 December 2010
}

CD5 is a transmembrane receptor expressed on $\mathrm{T}$ and B1a lymphocytes where it is known to modulate lymphocyte activation and/or differentiation processes. Recent evidence show that CD5 also works as pathogen recognition receptor for conserved fungal cell wall components ( $\beta$-glucans).

When analyzing SNP data in a worldwide sample of 39 human populations (HGDP diversity panel) different signatures of positive selection were detected in a $150 \mathrm{~kb}$ genomic region where the $C D 5$ gene maps. The Val471 to Ala substitution ( $r$ 22229177) in the cytoplasmatic region of $C D 5$ was suggested to be the most plausible target of selection as it was the only known nonsynonymous variant linked to the putatively selected haplotype. In silico analyses predict a possible deleterious function of Val to Ala replacement and one recent study reported better clinical prognosis for Valine in patients with chronic lymphocytic leukaemia (CLL). Resequencing analysis of the CD5 gene in 60 individuals representing three main populations (20 Africans, 20 Europeans and 20 Asians) has been performed in this study with the specific aims of fully describing its genetic variability and of confirming previous evidences of selection. The results obtained show a clear signal of positive selection at the CD5 locus in East Asians when applying classical tests of neutrality such as Tajima's $\mathrm{D}, \mathrm{Fu}$ and Li's F and D, or Fu and Wu's $\mathrm{H}$ and suggest that no other coding variations in this locus could be the actual target of selection. Interestingly, MAPK kinase assays performed on COS-7 cell transfectants and human peripheral blood lymphocytes subjected to different stimuli (anti-CD5 mAb crosslinking, Zymosan) demonstrated quantitative and qualitative functional differences

\footnotetext{
${ }^{1}$ Servei d'Immunologia, Hospital Clínic de Barcelona, IDIBAPS, Barcelona, Spain

Full list of author information is available at the end of the article
}

between the two allelic $C D 5$ variants. Taken together, the results strongly favor the hypothesis of a possible adaptive role of the V471A substitution in East Asian populations.

\begin{abstract}
Author details
'Servei d'Immunologia, Hospital Clínic de Barcelona, IDIBAPS, Barcelona, Spain. ${ }^{2}$ Institute of Evolutionary Biology (UPF-CSIC), CEXS - UPF - PRBB, Barcelona, Spain. ${ }^{3}$ Dept. de Biologia Cellular, Immunologia i Neurociències, UB, Barcelona, Spain.
\end{abstract}

Published: 25 November 2010

doi:10.1186/1479-5876-8-S1-P14

Cite this article as: Bielig et al:: Functional relevance of a non-

synonymous substitution in the CD5 gene (V471A) targeted for positive selection in East Asian populations. Journal of Translational Medicine 2010 8(Suppl 1):P14. 